\title{
Aplicación de la Escala de Depresión del Center of Epidemiological Studies en adolescentes de la Ciudad de México
}

\author{
Catalina González-Forteza, Dra en Psic Soc, (I) José Alberto Jiménez-Tapia, M en C, (I) \\ Luciana Ramos-Lira, Dra en Psic Soc, (1) Fernando A Wagner, D en C. ${ }^{(2)}$
}

\author{
González-Forteza C, Jiménez-Tapia JA, \\ Ramos-Lira L, Wagner FA. \\ Aplicación de la Escala \\ de Depresión del Center of Epidemiological Studies \\ en adolescentes de la Ciudad de México. \\ Salud Publica Mex 2008;50:292-299.
}

\section{Resumen}

Objetivo. Probar la validez de constructo, concurrente y externa, y la consistencia interna de la Escala de Depresión del Center for Epidemiologic Studies (CES-D-R) en adolescentes. Material y métodos. Estudio transversal con dos cohortes de estudiantes de secundaria del DF.El cuestionario incluyó la CES-D-R y otras escalas sobre problemas relacionados con suicidio, violencia, exposición a oportunidades y consumo de drogas. La participación fue voluntaria y anónima. Resultados. Se incluyó a I 549 estudiantes (edad promedio, I 4 años; $D E=1.2)$. La escala mostró una estructura de seis factores (varianza explicada, 55\%), consistencia interna excelente $(\alpha=0.93)$, discriminación significativa entre puntajes extremos $(z=-3.695, p<0.001)$ y correlación positiva y significativa con la escala de ideación suicida de Roberts $(r=0.685, p<0.00 \mathrm{I})$. Conclusiones. La CES-D-R tiene excelentes características psicométricas en adolescentes mexicanos, por lo que es adecuada para la detección oportuna de depresión. Esto permite utilizarla en las vigilancias sistemáticas y periódicas en la población escolar para detectar necesidades de atención, prevención y promoción de la salud mental.

Palabras clave: psicometría; escalas; estudiantes; depresión; adolescentes; México
González-Forteza C, Jiménez-Tapia JA, Ramos-Lira L, Wagner FA.

Application of the revised version

of the Center of Epidemiological Studies Depression Scale

in adolescent students from Mexico City.

Salud Publica Mex 2008;50:292-299.

\section{Abstract}

Objective. To assess the internal consistency, as well as the construct, concurrent and external consistency of the Center for Epidemiologic Studies Depression Scale (CES$\mathrm{D}-\mathrm{R}$ ) in Mexican adolescents. Material and Methods. The data are from two samples of middle-school students from Mexico City. The questionnaire included the CES-D-R and other scales for suicide problems, peer and family violence, and drug use. Results. The sample included I 549 students (mean age 14 years, $S D=1.2$ ). The CES-D-R showed a sixfactor structure (explained variance, $55 \%$ ) with an excellent internal consistency $(\alpha=0.93)$, a significant discriminative power for opposite scores $(z=-3.695, p<0.00 I)$, and a positive significant correlation with the Roberts Suicidal Ideation Scale $(r=0.685, p<0.00 I)$. Conclusions.The CES-D-R has excellent psychometric characteristics for Mexican adolescents and therefore is deemed as an adequate tool for the assessment of depressive symptoms in large samples to detect mental health needs and design preventive interventions.

Key words: psychometrics; scales; students; depression; adolescents; Mexico

(I) Instituto Nacional de Psiquiatría Ramón de la Fuente. México.

(2) Morgan State University. Baltimore, Estados Unidos de América.

Fecha de recibido: 30 de abril de 2007 - Fecha de aprobado: 13 de marzo de 2008 Solicitud de sobretiros: Dr. Fernando A. Wagner. Director, Prevention Sciences Research Center, Morgan State University. 1700 E. Cold Spring Lane, Portage Building, Suite 103. Baltimore, MD 2125I, USA. Email:fernando.wagner@morgan.edu 
L os trastornos mentales se consideran un problema de salud en casi todo el mundo; se calcula que para el año 2020 la depresión ocupará el segundo lugar como padecimiento incapacitante. ${ }^{1}$ Además, se considera que 20\% de los niños y adolescentes en el planeta sufre alguna enfermedad mental. En particular, los síntomas depresivos y la depresión durante la adolescencia se han convertido en problemas de una magnitud notoria, ${ }^{2}$ lo que genera una genuina preocupación en el sector salud dado su efecto presente y las consecuencias posteriores en la salud de los individuos. ${ }^{3}$

Diferentes estudios longitudinales en niños y adolescentes con trastorno depresivo mayor y distimia sugieren un riesgo elevado de episodios depresivos recurrentes vinculados con suicidios y otros comportamientos autodestructivos que inician en esta etapa y pueden prolongarse hasta la edad adulta. ${ }^{4,5}$

Debido a la gran cantidad de cambios y procesos que ocurren durante la adolescencia, ésta se convierte en una etapa de vida en la que puede aumentar la exposición a situaciones que resultan difíciles de afrontar para los individuos y, en consecuencia, se vuelven fuentes de estrés; por ello es conveniente analizarla de manera cuidadosa para distinguir entre las expresiones afectivas, cognitivas, somáticas y conductuales atribuibles al periodo y las que pueden relacionarse con un malestar y que tendría consecuencias en la salud emocional a corto y mediano plazos. ${ }^{6,7}$

Los profesionales de la salud mental enfrentan la necesidad de estudiar el curso de los síntomas depresivos en este periodo de la vida, ya que -entendidos como un componente de la depresión- influyen sobre el estado de ánimo y las situaciones vividas como poco placenteras o molestas, lo cual los convierte en un factor importante de vulnerabilidad en el estado emocional de los adolescentes. Cabe recordar que la depresión afecta todas las áreas de la vida de esta población, además de que incrementa la probabilidad de ocurrencia de otros trastornos psiquiátricos y conductas problemáticas. ${ }^{8,9}$ Asimismo, el estudio de la depresión en la adolescencia muestra aspectos todavía poco estudiados, como las diferencias y similitudes entre hombres y mujeres desde la perspectiva de género. ${ }^{8}$

En virtud de la magnitud y la tendencia crecientes de la depresión en la población adolescente y las dificultades concomitantes para la salud de estos individuos y para el propio sistema de salud, ${ }^{2}$ es prioritario enfocar los esfuerzos en la detección oportuna y la prevención del padecimiento para reducir los riesgos en el desarrollo psicosocial.

En este sentido, los instrumentos de tamizaje válidos y confiables tienen una gran utilidad práctica y metodológica porque proporcionan una aproximación precisa para la detección de posibles casos clínicos de depresión. Radloff ${ }^{10}$ diseñó la Escala de Depresión del Centro de Estudios Epidemiológicos (Center for Epidemiologic Studies, Depression Scale: CES-D) para disponer de una herramienta que satisficiera estas características. Sin embargo, cabe destacar que la versión original de la CES-D se ideó muchos años antes de que se contara con los criterios diagnósticos y clínicos incorporados en la décima Clasificación de las Enfermedades de la Organización Mundial de la Salud o la cuarta edición del Manual Diagnóstico y Estadístico de la Asociación Psiquiátrica Americana (DSM-IV).${ }^{11}$ Debido al carácter dinámico de los constructos teóricos y las poblaciones, el Centro para la Investigación de Prevención de la Universidad Johns Hopkins se propuso actualizar y compatibilizar los contenidos de tamizaje de la CES-D con los criterios diagnósticos para episodios de depresión mayor (EDM), propuestos en el DSM-IV,11 lo que resultó en una versión revisada: la CES-D-R. ${ }^{12}$ La primera tarea consistió en analizar la convergencia entre la versión original de la CES-D y los criterios del DSM-IV; de ese modo pudo observarse que se sobrevaloraba la presencia de síntomas de disforia y, en cambio, no se incluían suficientes reactivos para valorar la presencia de problemas de peso, sueño, autoestima y concentración, y ninguno respecto del retardo psicomotor, la agitación y la ideación suicida. ${ }^{12}$ Tales disparidades pueden tener implicaciones de salud pública, sea porque se incorpora un número excesivo de posibles casos al incluirse síntomas relacionados con la tristeza y el retraimiento, que no son indicativos de depresión en grado suficiente, sea porque no se reconoce el posible curso de depresión en personas con expresiones leves de tristeza o retraimiento, acompañadas de diversos síntomas que no se valoran en la escala original de la CES-D.

En virtud de que la CES-D se ha utilizado de forma satisfactoria en la población adolescente mexicana, ${ }_{13}^{13-16}$ y de que un estudio piloto proporcionó datos muy favorables en cuanto a la utilidad del CES-D-R con población de edad avanzada, ${ }^{17,18}$ ha surgido la necesidad de evaluar las características psicométricas de esta nueva escala con adolescentes mexicanos. Este trabajo tiene como objetivos probar la validez del constructo y la consistencia interna de la escala y sus dimensiones, su validez concurrente por medio de grupos contrastados y su validez externa en adolescentes estudiantes.

\section{Material y métodos}

Los datos se recabaron de todos los estudiantes inscritos en dos escuelas de educación media, ubicadas en el 
centro histórico de la Ciudad de México, durante los ciclos escolares de 2003 y 2006. Los planteles escolares participantes se seleccionaron por su contraste: uno con el mayor índice de aprobación y el otro con el mayor índice de reprobación, según los indicadores académicos de la Secretaría de Educación Pública. ${ }^{17}$

\section{Participantes}

La muestra global quedó conformada con 1549 estudiantes, con una media de edad de 14 años $(D E=1.2)$; $54 \%$ se integró con hombres y $46 \%$ con mujeres; $83 \%$ informó que fueron estudiantes de tiempo completo la mayor parte del año anterior al estudio y que no trabajaron con sueldo devengado (84\%). La mayoría $(81 \%)$ tenía padre/figura paterna y $95 \%$ madre/ figura materna.

\section{Instrumento}

Se diseñó un cuestionario que incluyó la CES-D-R y otras secciones para evaluar e indagar en relación con la ideación y el intento suicida, la violencia (familiar y de pares), la exposición a oportunidades para consumir drogas y su consumo. ${ }^{17}$

El proceso de revisión de la CES-D-R quedó integrado por diferentes etapas..$^{18} \mathrm{~A}$ los 20 reactivos de la versión original se agregaron 15 , los cuales pasaron por un proceso de traducción, adaptación y análisis de correlación con criterios diagnósticos. ${ }^{12,18}$ La traducción y adaptación de reactivos del inglés al español se efectuó en grupo mediante discusiones en las que participaron profesionales de la psicología y la epidemiología. Se retomaron los reactivos de la versión original de la CES-D, que ya se habían traducido y probado. ${ }^{14,15}$ Los nuevos se tradujeron ajustados al mismo estilo de la escala original (p. ej., tiempo y persona en cada pregunta). La armonización incluyó la traducción y discusión acerca de la concordancia entre los reactivos y los criterios diagnósticos del DSM-IV, lo que llevó a introducir adecuaciones para mejorar la concordancia con los criterios diagnósticos y la clasificación que publicaron de modo original Eaton y colaboradores. ${ }^{12}$

Los nuevos reactivos evalúan lo siguiente: ánimo deprimido (un reactivo), cambios drásticos en el peso (un reactivo); tipo de pensamiento (un reactivo); fatiga (un reactivo); agitación o retardo psicomotor (dos reactivos); ideación suicida (dos reactivos); problemas de sueño (dos reactivos); culpa excesiva o inapropiada (dos reactivos); y disminución del placer (tres reactivos).

Además de los reactivos, se incluyó una opción de respuesta que amplía los límites temporales a las últimas dos semanas, con lo que se cubren los criterios del DSMIV en cuanto a la duración mínima de los síntomas para cumplir un diagnóstico.

Por último, se diseñó un algoritmo que incorpora los criterios diagnósticos y que permite valorar la presencia y frecuencia de los síntomas ${ }^{18}$ a través de cinco categorías de clasificación mutuamente excluyentes:

1. Sintomas de EDM clínicamente relevantes, presencia de síntomas en al menos cinco criterios, incluidos los síntomas de anhedonia o disforia durante dos semanas.

2. EDM altamente probable, presencia de anhedonia o disforia durante casi todos los días en las últimas dos semanas, pero con sólo cuatro síntomas (uno menos que el criterio del DSM-IV).

3. EDM probable, presencia de anhedonia o disforia durante casi todos los días en las últimas dos semanas pero con sólo tres síntomas (dos menos que el criterio del DSM-IV).

4. Episodio depresivo subumbral, indicado por un resultado de 16 o más puntos en la escala revisada y sin figurar en alguna de las categorías anteriores.

5. Ausencia de sintomas de EDM de relevancia clínica, incluidos los individuos que tuvieron un resultado menor a 16 puntos en la escala revisada. El puntaje de la escala se obtiene tras sumar los reactivos por síntoma del DSM-IV y aplicar un proceso de ponderación para tener los mismos límites y distribución que los de la escala original del CES-D, es decir, el umbral de 16 puntos que suele utilizarse para determinar la presencia o la ausencia de síntomas depresivos.

\section{Procedimiento y consideraciones éticas}

Se estableció contacto con autoridades de la SEP y los planteles para obtener los permisos correspondientes. Los padres o tutores de los alumnos, así como estos mismos, recibieron un formato escrito de consentimiento informado en el que se explicaban las condiciones de su inclusión en el estudio, la participación voluntaria y el carácter anónimo del registro de la información. El comité de ética del Instituto Nacional de Psiquiatría Ramón de la Fuente Muñiz revisó y aprobó tanto el proyecto como los formatos de consentimiento. La recolección de datos se efectuó en los salones de clase, una vez que los estudiantes aceptaron contestar el cuestionario, y recibieron el instrumento en formato autoaplicable de lápiz-papel. No se presentaron rechazos. La aplicación la llevó a cabo un equipo de psicólogos en formación (pasantes), previo curso de capacitación y entrenamiento 
y sin la presencia de los maestros ni de otra autoridad escolar. Para la captura y procesamiento de datos se usó la versión 13.0 de SPSS.*

\section{Resultados}

\section{Validez del constructo y consistencia interna}

Se realizó un análisis para cuantificar la consistencia interna de la escala global, la cual mostró un índice muy satisfactorio $(\alpha=0.93)$. No se eliminó ninguno de los 35 reactivos; el criterio principal para conservarlos fue la homogeneidad del coeficiente de confiabilidad observado si el ítem fuera removido, y la correlación con el puntaje total y la covarianza promedio de la escala, con cada ítem y sin él. Los análisis por subgrupos de edad y sexo mostraron coeficientes de consistencia interna aceptables, según se muestra en el cuadro I.

Como puede observarse, el coeficiente de consistencia interna fue mayor entre las mujeres. No obstante que los índices de confiabilidad son adecuados para todos y cada uno de los grupos de edad, se puede advertir que los hombres más jóvenes tuvieron los índices menores.

Se realizó un análisis factorial con extracción de componentes principales y rotación oblicua (ya que se esperaba que las variables tuvieran una gran correlación entre sí), el cual arrojó una estructura de seis factores en 20 iteraciones, con valores Eigen mayores a uno, que explican $54.61 \%$ de la varianza. La consistencia interna y la concordancia teórica de éstos resultaron satisfactorias. Los factores fueron los siguientes: a) afecto deprimido, b) afecto positivo, c) inseguridad emocional y problemas interpersonales, d) somatización, e) bienestar emocional y f) actividad retardada (cuadro II). Cabe señalar que el reactivo 21: "Me molesté por cosas que usualmente no me molestan", se eliminó debido a que no tuvo un peso factorial $\geq .30$.

\section{Validez concurrente por medio de grupos contrastados}

Se realizó una prueba de grupos contrastados y se compararon los puntajes globales de la CES-D-R que se situaron por debajo del percentil 25, en comparación

\footnotetext{
* SPSS para Windows. Versión 13.0. Illinois, USA: SPSS INC, 2004.
}

\begin{tabular}{|c|c|c|}
\hline \multicolumn{3}{|c|}{ Cuadro I } \\
\hline \multicolumn{3}{|c|}{ COEFICIENTE DE CONSISTENCIA INTERNA } \\
\hline \multirow{3}{*}{\multicolumn{3}{|c|}{$\begin{array}{l}\text { DE LA CES-D-R EN ADOLESCENTES ESTUDIANTES } \\
\text { DE SECUNDARIA DE LA CIUDAD DE MÉXICO (N=I 52I) } \\
\text { POR GRUPOS DE EDAD Y SEXO. } 2003 \text { Y } 2006\end{array}$}} \\
\hline & & \\
\hline & & \\
\hline & \multicolumn{2}{|c|}{ Alfa de Cronbach } \\
\hline ad & $\begin{array}{c}\text { Hombres } \\
(n=832)\end{array}$ & $\begin{array}{l}\text { Mujeres } \\
(n=689)\end{array}$ \\
\hline 2 años o menos & 0.87 & 0.93 \\
\hline 3 años & 0.91 & 0.94 \\
\hline 4 años & 0.90 & 0.94 \\
\hline 5 o más años & 0.92 & 0.95 \\
\hline
\end{tabular}

con aquéllos por arriba del percentil 75. Se utilizó una comparación de promedios de Mann-Whitney, la cual mostró diferencias estadísticamente significativas entre los cuartiles contrastados $(z=-3.695, p<0.001)$, lo que indica que la escala discrimina de modo adecuado entre puntajes extremos.

\section{Validez externa}

Para la validez externa se llevó a cabo una prueba de asociación entre la CES-D-R y la escala de ideación suicida de Roberts, ${ }_{19}^{19}$ en la cual se identificó una correlación positiva y significativa (Pearson $\mathrm{r}=0.685, p<0.001$ ).

Por último, se aplicaron los algoritmos provisionales que se elaboraron y cuyos umbrales se encuentran en proceso de validación clínica. Con estos puntos en consideración, se determinó que $8.2 \%$ de los estudiantes de esta muestra cursaba un episodio de depresión mayor, con un porcentaje significativamente mayor para las mujeres $(13.5 \%)$ respecto de los hombres $(3.8 \%)(\chi 2=$ 46.7, $\mathrm{p}<0.0001$ ) (cuadro III).

De este modo, si se agregan los porcentajes de las frecuencias totales de probable episodio de depresión mayor, se puede observar que $11.7 \%$ de los hombres y 12.9\% de las mujeres presentaron síntomas notorios de depresión que no rebasan el umbral clínico necesario para establecer un diagnóstico de episodio mayor, pero que pueden indicar la presencia de problemas emocionales de importancia. De la misma manera, al sumar los porcentajes de los sujetos que presentaron signos relevantes de problemas depresivos, se calculó que una de cada cuatro mujeres $(26.4 \%$ ) y uno de cada siete hombres $(15.5 \%)$ tenían síntomas significativos de depresión. 


\section{Cuadro II}

\section{Estructura FACTORIAL DE LA CES-D-R EN ADOLESCENTES ESTUDIANTES DE SECUNDARIA. Ciudad de México, 2003 y 2006}

Peso factorial \% de varianza explicada Alfa de Cronbach

Afecto deprimido

30.05

0.90

2. No podía quitarme la tristeza

0.785

6. Me sentía triste

0.727

4. Me sentía deprimido(a)

0.669

14. Sentía deseos de estar muerto(a)

0.360

9. Sentía que era una mala persona

0.336

Afecto positivo

7.99

0.80

$\begin{array}{ll}\text { 31. Disfruté la vida } & 0.847 \\ \text { 33. Me divertí mucho } & 0.838 \\ \text { 27. Me sentía feliz } & 0.790\end{array}$

Inseguridad emocional y problemas interpersonales

4.16

0.94

\begin{tabular}{ll}
\hline 26. Me sentía temeroso(a) & 0.715 \\
\hline 32. Tenía ataques de llanto & 0.658 \\
\hline 30. Las personas eran poco amigables & 0.643 \\
\hline 34. Sentía que iba a darme por vencido(a) & 0.633 \\
\hline 25. Pensé que mi vida había sido un fracaso & 0.541 \\
\hline 29. Me sentía solo(a) & 0.532 \\
\hline 35. Sentía que le desagradaba a la gente & 0.522 \\
\hline 28. Hablé menos de lo usual & 0.408 \\
\hline 17. Estaba a disgusto conmigo mismo(a) & 0.326 \\
\hline 15. Quería hacerme daño & 0.32 I
\end{tabular}

Somatización

3.67

0.91

\begin{tabular}{ll} 
19. Me costaba mucho trabajo dormir & 0.619 \\
\hline 5. Dormía sin descansar & $0.57 \mid$ \\
\hline 16. Me sentía cansado(a) todo el tiempo & 0.563 \\
\hline 3. Tenía dificultad para mantener mi mente en lo que estaba haciendo & 0.520 \\
I. Tenía poco apetito & 0.513 \\
18. Perdí peso sin intentarlo & 0.460 \\
20. Era difícil concentrarme en las cosas importantes & 0.378
\end{tabular}

Bienestar emocional

3.57

0.81

23. Sentí que todo lo que hacía era con esfuerzo

0.838

22. Sentía que era tan bueno(a) como otra gente

$-0.758$

24. Me sentía esperanzado(a) hacia el futuro

$-0.620$

Actividad retardada

3.16

0.92

12. Sentía que me movía muy lento

$-0.712$

13. Me sentía agitado(a)

$-0.663$

II. Dormía más de lo habitual

$-0.611$

10. Había perdido interés en mis actividades diarias

$-0.488$

7. No podía seguir adelante

$-0.44$

8. Nada me hacía feliz

$-0.332$ 
Cuadro III

SínTOMAS DE EPISOdIOS DE DEPRESIÓN MAYOR ENTRE ESTUDIANTES MEXICANOS DE ENSEÑANZA MEDIA de la Ciudad de MéXico por SeXo ( $=$ = 504*). 2003 y 2006

\begin{tabular}{|c|c|c|c|c|c|c|}
\hline \multirow[b]{2}{*}{ Clasificación según CES-D-R } & \multicolumn{2}{|c|}{ Frecuencias } & \multicolumn{2}{|c|}{ Hombres } & \multicolumn{2}{|c|}{ Mujeres } \\
\hline & Total & $\%$ & \# & $\%$ & \# & $\%$ \\
\hline Sin relevancia clínica & 723 & 48.0 & 471 & 57.2 & 252 & 37.0 \\
\hline Depresión subumbral & 474 & 31.5 & 225 & 27.3 & 249 & 36.6 \\
\hline Probable DSM-IV EDM & 64 & 4.3 & 40 & 4.9 & 24 & 3.5 \\
\hline Altamente probable DSM-IV EDM & 120 & 8.0 & 56 & 6.8 & 64 & 9.4 \\
\hline EDM (DSM-IV) & 123 & 8.2 & 31 & 3.8 & 92 & 13.5 \\
\hline Total & I 504 & 100 & 823 & 100 & 681 & 100 \\
\hline $\begin{array}{l}\text { H: hombres } \\
\text { M: mujeres }\end{array}$ & & & & & & \\
\hline EDM: episodio de depresión mayor & & & & & & \\
\hline
\end{tabular}

\section{Discusión}

La Escala de Depresión del Centro de Estudios Epidemiológicos ha probado su validez y confiabilidad en diferentes poblaciones mexicanas. ${ }^{14,15,20-22} \mathrm{Sin}$ embargo, la versión revisada ofrece ventajas sobre la original porque corresponde mejor a los sistemas contemporáneos de clasificación de las enfermedades, satisface de manera más amplia las diversas dimensiones de los síntomas depresivos y obtiene valoraciones de mayor confiabilidad para cada uno de ellos dado que cuenta al menos con tres reactivos para cada síntoma. Cabe hacer notar que los resultados del presente estudio indican que la versión revisada de la escala CES-D-R mantiene las características psicométricas que la convirtieron en una de las escalas más usadas en el mundo para la valoración de la depresión en estudios poblacionales con adolescentes y personas de edad avanzada. Asimismo, puesto que la versión revisada contiene en su totalidad los reactivos de la versión original, y si se considera que se ha creado un algoritmo especial, es posible comparar los resultados de ambas escalas. ${ }^{18}$

Una de las particularidades de esta nueva versión de la escala es que puede emplearse como un instrumento de tamizaje para la detección oportuna de casos probables de depresión, o bien como un instrumento diagnóstico confiable para evaluar síntomas que cumplan con los criterios del DSM-IV. Sin embargo, es necesario realizar evaluaciones posteriores del instrumento en trabajos en los que se incluyan muestras clínicas para cimentar aún mejor su validez y confiabilidad.

Ahora bien, es difícil comparar los resultados obtenidos en el presente estudio con otras investigaciones por dos motivos principales: el lapso de tiempo utilizado para calcular las prevalencias de EDM y la diversidad de poblaciones estudiadas. En la mayoría de los artículos se notifican tasas de EDM "alguna vez en la vida" o en el "último año" y además las muestras probabilísticas son en particular de personas de 18 años y más en comparación con las de estudiantes ubicados en una zona de alto riesgo como la del presente estudio. ${ }^{23,24} \mathrm{No}$ obstante, puede advertirse que la prevalencia de EDM en las "últimas dos semanas" aquí registrada (3.5\%) para los hombres es similar a la tasa anual encontrada en dos estudios con muestras probabilísticas con adultos mexicanos, ${ }^{25,26}$ mientras que la tasa de EDM entre las estudiantes mujeres de este estudio $(13.5 \%)$ prácticamente duplica la correspondiente a la prevalencia anual informada en dichos estudios para esa población.

Esta nueva versión revisada significa un buen avance en términos de tamizaje, ya que incorpora una mayor compatibilidad con los criterios diagnósticos aceptados; los reactivos pasaron por un proceso completo de adaptación para satisfacer los requerimientos clínicos $^{12}$ y para el contexto mexicano. Además, la estructura conceptual, la consistencia interna y el valor discriminante resultaron bastante satisfactorios.

Cabe resaltar que este instrumento es muy conveniente por su brevedad y porque no requiere personal especializado para su aplicación y calificación, lo que representa un bajo costo y un elevado beneficio, ya que puede utilizarse en contextos ajenos a la práctica clínica psiquiátrica: estudios con población general, grupos escolares, centros de reclusión, albergues temporales y el primer nivel de atención.

De cualquier manera, es necesario continuar el desarrollo y la depuración de instrumentos que funcionen para detectar de manera oportuna a individuos (en 
este caso adolescentes) que son proclives a desarrollar algún tipo de problemática en su salud mental. Esto posibilitaría la aplicación de mecanismos de prevención secundaria más confiables para reducir el efecto de malestares y padecimientos mentales a corto y mediano plazos.

Los resultados de este trabajo muestran que la CES-D-R es un instrumento que posee características psicométricas satisfactorias de confiabilidad y validez para adolescentes estudiantes mexicanos, razón por la cual es posible considerarla como una herramienta útil y adecuada para la detección oportuna de depresión, además de que su aplicación es fácil. Su utilización en vigilancias sistemáticas y periódicas en la población escolar podría traducirse en programas adecuados de prevención y promoción de la salud mental en los adolescentes.

\section{Agradecimientos}

Se agradece al Conacyt el financiamiento otorgado a los proyectos 25902 "Intentos de suicidio y violencia intrafamiliar: aspectos culturales, prevalencia, factores asociados y percepción subjetiva en adolescentes" y 44915 "Hacia la prevención de la depresión y del riesgo suicida: uso de servicios, detección y estrategia psicoeducativa en estudiantes de secundaria en el Centro Histórico de la Ciudad de México". El Doctor Wagner recibió apoyo del National Institute on Drug Abuse, grants DA012390 y DA019805, así como del National Center on Minority Health and Health Disparities, grant MD000217.

\section{Referencias}

I. Murray C, Lopez A. Alternative projections of mortality and disability by cause, 1990-2020: Global Burden of Disease Study. Lancet 1997;349: |498-| 504.

2. Benjet C, Borges G, Medina-Mora ME, Fleiz C, Zambrano J. La depresión con inicio temprano: prevalencia, curso natural y latencia para buscar tratamiento. Salud Publica Mex 2004;46(5):417-423.

3. Franko D, Striegel-Moore R, Bean J, Tamer R, Kraemer H, Dohm F,et al. Psychosocial and health consequences of adolescent depression in black and white young adult women. Health Psychol 2005;24(6):586-593. 4.Weisman M,Wolf S, Golsdatein R. Depressed adolescents grown up. JAMA 1999;218(18):1707-17/3.

5. Giaconia R, Reinherz H, Paradis A, Carmola-Hauf A, Stashwick C. Major depression and drug disorders in adolescence: general and specific impairments in early adulthood. J Am Acad Child and Adolesc Psychiatry 200I;40(I2):I426-I433.
6. Macías G. Psicoterapia del niño y del adolescente deprimidos. Salud Ment 1985;8(4):3-7.

7. De la Fuente R, Medina-Mora ME, Caraveo J. Salud mental en México. México: Fondo de Cultura Económica, 1997.

8. Marmorstein N, lacono W. Mayor depression and conduct disorder in a twin sample: gender, functioning and risk for future psychopathology. J Am Acad Child Adolesc Psychiatry 2003;42(2):225-233.

9. Birhamer B,Williamson D, Dahl R,Axelson D, Kaufman J, Dorn L, et al. Clinical presentation and course of depression in youth: Does onset in childhood differ form onset in adolescence? J Am Acad Child Adolesc Psychiatry 2004;43(I):63-70.

I0. Radloff L.The CES-D Scale: a self report depression scale for research in the general population. Appl Psychological Measurement 1977; I:385-40I. I I.American Psychiatric Association. DSM-IV. Barcelona: Masson, 1997. 12. Eaton W, Muntaner C, Smith C. Revision of the Center of Epidemiological Studies Depression (CES-D) Scale. Baltimore: Johns Hopkins University Prevention Center, 1998.

13. González-Forteza C,Andrade P, Jiménez A. Estresores cotidianos familiares, sintomatología depresiva e ideación suicida en adolescentes mexicanos. Acta Psiquiátrica Psicol Am Lat 1997;43(4):319-326. 14. Benjet C, Hernández L, Tercero G, Hernández A, Chartt R. Validez y confiabilidad de la CES-D en peri-púberes. Rev Mex Psicol 1999; I6(I):175-185.

15. Aguilera R, Carreño S, Juárez F. Características psicométricas de la CES-D en una muestra de adolescentes rurales mexicanos de zonas con alta tradición migratoria. Salud Ment 2004;27(6):57-66.

16. Caballero M, Ramos L, González-Forteza C, Saltijeral M. La violencia que ejercen los padres hacia sus hijos adolescentes. En: Jiménez M, ed. Caras de la violencia familiar. México: UNAM, 2005:259-272.

17.Wagner F, González-Forteza C,Aguilera R, Ramos L, MedinaMora ME,Anthony J. Oportunidades de exposición al uso de drogas entre estudiantes de secundaria de la Ciudad de México. Salud Ment 2003;26(2):22-32.

18. Reyes M, Soto A, Milla J, García-Vázquez A, Hubard L, Mendoza H,et al.Actualización de la Escala de Depresión del Centro de Estudios Epidemiológicos (CES-D). Estudio piloto en una muestra geriátrica mexicana. Salud Ment 2003;26(I):59-68.

19. Roberts E. Reliability of the CES-D Scale in different ethnic contexts. Psychiatry Research 1980;2:125-I34.

20. Mariño MC, Medina-Mora ME, Chaparro J, González-Forteza C. Confiabilidad y estructura factorial del CES-D en una muestra de adolescentes mexicanos. Rev Mex Psicol 1993; I0(2):I4I-145.

2I. Salgado N, Maldonado M. Características psicométricas de la Escala de Depresión del Centro de Estudios Epidemiológicos en mujeres mexicanas adultas de áreas rurales. Salud Publica Mex 1994;36(2):200-209.

22. Vera J. Evaluación del inventario de depresión (CES-D) en mujeres de la zona rural del norte de México. En: La Psicología Social en México. México:AMEPSO, 1996:496-50I.

23. González-Forteza C, Ramos-Lira L, Caballero-Gutiérrez MA,Wagner FA. Correlatos psicosociales de depresión, ideación e intento suicida en adolescentes mexicanos. Psicothema 2003; 15(4): 524-532.

24. Ramos-Lira L, Gonzalez-Forteza C,Wagner FA.Violent victimization and drug involvement among mexican middle school students. Addiction 2006; I0I (6):850-856.

25. Belló M, Puentes-Rosas E, Medina-Mora ME, Lozano R. Prevalencia y diagnóstico de la depresión en Mexico. Salud Publica Mex 2005;47(Supl I):S4-SII.

26. Slone LB, Norris FH, Murphy AD, Baker CK, Perilla JL, Diaz D,et al. Epidemiology of major depression in four cities in Mexico. Depress Anxiety 2006;23(3):158-167. 
Anexo

\section{Versión REVISAdA de LA CES-D-R}

A continuación hay una lista de emociones y situaciones que probablemente hayas sentido o tenido. Por favor escribe durante cuántos días en la semana pasada te sentiste así, o si te ocurrió casi diario en las últimas dos semanas

\begin{tabular}{|c|c|c|c|c|c|}
\hline & & En las & ana pasada & & Últimas dos semanas \\
\hline Jurante cuántos días... & $\begin{array}{l}\text { Escasamente } \\
\text { (0 a I dias) }\end{array}$ & $\begin{array}{c}\text { Algo } \\
\text { (I a } 2 \text { días) }\end{array}$ & $\begin{array}{l}\text { Ocasionalmente } \\
\text { (3 a } 4 \text { dias) }\end{array}$ & $\begin{array}{l}\text { La mayoría } \\
\text { (5 a } 7 \text { dias) }\end{array}$ & $\begin{array}{l}\text { Casi diario } \\
\text { (10 a l4 días) }\end{array}$ \\
\hline
\end{tabular}

\begin{tabular}{|c|c|c|c|c|c|}
\hline I. Tenía poco apetito & 0 & I & 2 & 3 & 4 \\
\hline 2. No podía quitarme la tristeza & 0 & I & 2 & 3 & 4 \\
\hline 3. Tenía dificultad para mantener mi mente en lo que estaba haciendo & 0 & I & 2 & 3 & 4 \\
\hline 4. Me sentía deprimido(a) & 0 & 1 & 2 & 3 & 4 \\
\hline 5. Dormía sin descansar & 0 & 1 & 2 & 3 & 4 \\
\hline 6. Me sentía triste & 0 & I & 2 & 3 & 4 \\
\hline 7. No podía seguir adelante & 0 & 1 & 2 & 3 & 4 \\
\hline 8. Nada me hacía feliz & 0 & I & 2 & 3 & 4 \\
\hline 9. Sentía que era una mala persona & 0 & 1 & 2 & 3 & 4 \\
\hline 10. Había perdido interés en mis actividades diarias & 0 & 1 & 2 & 3 & 4 \\
\hline II. Dormía más de lo habitual & 0 & I & 2 & 3 & 4 \\
\hline 12. Sentía que me movía muy lento & 0 & 1 & 2 & 3 & 4 \\
\hline 13. Me sentía agitado(a) & 0 & 1 & 2 & 3 & 4 \\
\hline 14. Sentía deseos de estar muerto(a) & 0 & I & 2 & 3 & 4 \\
\hline 15. Quería hacerme daño & 0 & 1 & 2 & 3 & 4 \\
\hline 16. Me sentía cansado(a) todo el tiempo & 0 & 1 & 2 & 3 & 4 \\
\hline 17. Estaba a disgusto conmigo mismo(a) & 0 & 1 & 2 & 3 & 4 \\
\hline 18. Perdí peso sin intentarlo & 0 & 1 & 2 & 3 & 4 \\
\hline 19. Me costaba mucho trabajo dormir & 0 & 1 & 2 & 3 & 4 \\
\hline 20. Era difícil concentrarme en las cosas importantes & 0 & 1 & 2 & 3 & 4 \\
\hline 21. Me molesté por cosas que usualmente no me molestan & 0 & I & 2 & 3 & 4 \\
\hline 22. Sentía que era tan bueno(a) como otra gente & 0 & I & 2 & 3 & 4 \\
\hline 23. Sentí que todo lo que hacía era con esfuerzo & 0 & 1 & 2 & 3 & 4 \\
\hline 24. Me sentía esperanzado(a) hacia el futuro & 0 & 1 & 2 & 3 & 4 \\
\hline 25. Pensé que mi vida ha sido un fracaso & 0 & 1 & 2 & 3 & 4 \\
\hline 26. Me sentía temeroso(a) & 0 & 1 & 2 & 3 & 4 \\
\hline 27. Me sentía feliz & 0 & 1 & 2 & 3 & 4 \\
\hline 28. Hablé menos de lo usual & 0 & I & 2 & 3 & 4 \\
\hline 29. Me sentía solo(a) & 0 & 1 & 2 & 3 & 4 \\
\hline 30. Las personas eran poco amigables & 0 & 1 & 2 & 3 & 4 \\
\hline 31. Disfruté de la vida & 0 & 1 & 2 & 3 & 4 \\
\hline 32. Tenía ataques de llanto & 0 & 1 & 2 & 3 & 4 \\
\hline 33. Me divertí mucho & 0 & I & 2 & 3 & 4 \\
\hline 34. Sentía que iba a darme por vencido(a) & 0 & 1 & 2 & 3 & 4 \\
\hline 35. Sentía que le desagradaba a la gente & 0 & I & 2 & 3 & 4 \\
\hline
\end{tabular}

